\title{
AIDS: trends, predictions, controversy
}

\author{
RoyAnderson
}

One of the persistent controversles that surfaces in the medla about AIDS Is whether the heterosexual population is at risk. The latest projections provide an emphatic affirmative.

PEOPLE working in AIDS research may be feeling somewhat besieged at present with frequent press comment on controversies such as the potential magnitude of the epidemic in developed countries and on the frustratingly slow progress in drug and vaccine development. The lack of balance in reports in some sectors of the press and media has not helped, with a particular trend in recent coverage of AIDS being the assertion that epidemiologists have greatly exaggerated the potential of the virus to spread in heterosexual populations in developed countries, and even a denial that there is a major HIV epidemic in sub-Saharan Africa despite, in the latter case, numerous studies that chart the steady rise of infection in low- and high-risk groups in urban centres and increasingly in rural areas in these regions 1 . The extreme views presented by some, in particular the Sunday Times in giving generous space to the Duesberg sect, have been balanced by careful and accurate reporting by others, notably the New York Times. But the diversity of views presented has created confusion in the minds of the public, researchers in other fields, and policy makers.

What is the current pattern of spread of AIDS in industrialized countries? Many of them have sophisticated surveillance systems that reveal the temporal evolution in the incidence of AIDS within different at-risk groups. The median incubation period of AIDS (time from infection to the development of AIDS) appears to be approximately 10 years in sexually active adults in developed countries irrespective of risk group, hence reports of the current incidence of AIDS reveal the pattern of HIV spread a decade ago. To improve the information base available to planners and policy makers, many countries have developed unlinked anonymous monitoring of HIV prevalence among people attending medical clinics ${ }^{2}$.

In Europe, temporal trends in the incidence of AIDS in most countries depict a steady rise over the past decade (see Fig. $1)^{3}$. But there are important differences between countries in the overall magnitude of the epidemic and its constituent pattern in different at-risk groups. For example, cumulative AIDS cases per million head of population at the end of 1992 were approximately 1,200 in the United States, 441 in Spain, 417 in Switzerland, 403 in France, 272 in Italy, 120 in the United Kingdom and 89 in Sweden ${ }^{3}$. For the last year for which accurate data are available (1991-92) in Europe as a whole the largest percentage increase occurred in the heterosexual transmission group (24.8), although there is much variability between countries. In the United States and the United Kingdom, cases in homosexual and bisexual men form about $60-70 \%$ of the total, but cases among heterosexuals now exceed those among intravenous drug users to form the

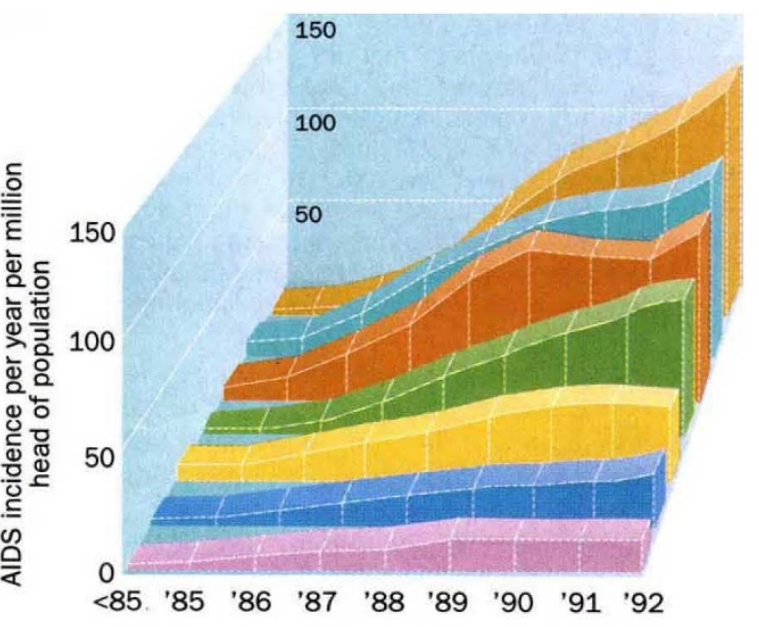

Spain $=$ France Switzerland

Italy Denmark UK Un Sweden

FIG. 1 Reported incidence of AIDS per annum, corrected for reporting delay, in a selection of European countries (data from ref. 13).

second-highest risk category. Of the new reports of AIDS in the United Kingdom during March 1993, for example, $60 \%$ were in homosexual or bisexual men, $15 \%$ in heterosexuals and $9 \%$ in injecting drug users. Conversely, in southern European countries such as Italy and Spain, injecting drug use is the major risk. Even within countries, however, the pattern varies ${ }^{2}$. Trends in HIV seroprevalence are more difficult to obtain but reports from a European study ${ }^{4}$ of patients attending sexually transmitted disease clinics revealed wide variation. Among nonintravenous drug using heterosexuals, prevalence ranged from 1-2\% in France, Germany, Italy, Spain and Switzerland, to $0-0.3 \%$ in the United Kingdom, Sweden and Denmark.

There are several general conclusions that can tentatively be drawn from these observed patterns. The epidemic is still in its early stages in most risk groups, although there are encouraging signs of a slowing in the rate of accumulation of new AIDS case reports in homosexual/ bisexual men in most developed countries. The long duration of the epidemic is a simple consequence of the long average incubation period of AIDS. Its full extent will not be known for many decades. This point has been made numerous times over the past 7 years $^{3}$, but the message of a slowly developing epidemic, particularly in the lower risk groups (heterosexuals), is still not accurately reported in many sectors of the press. To assess the likelihood of a more widely disseminated epidemic among heterosexuals, the important point is to base this assessment not on the comparatively low numbers of AIDS cases in this group at present, but on the trends in AIDS incidence and HIV seroprevalence over the past decade. These reveal an expanding epidemic, moving on a very slow timescale, largely centred at present in urban centres in poor communities and in socially marginalized groups.

There remain many uncertainties about the key epidemiological determinants of the epidemic. These include the likely pattern of the full incubation period distribution of AIDS; the fraction of those infected who will eventually develop AIDS; fluctuations in infectiousness during the incubation period; the relevance to transmission and pathogenesis of the genetic diversity of the virus; the prevailing heterogeneities in, and frequencies of, the behaviours that determine transmission; patterns of mixing or contact within and between the different at-risk groups; and the influence of education on these behaviours and mixing patterns. These uncertainties will not be resolved quickly.

Projections of future trends are obviously required, despite these uncertainties, to plan sensibly for health-care needs and to assess priorities in expenditure. Projections can only be made in quantitative terms for the short term $(2-4$ years). The methods available include extrapolation on the basis of simple mathematical functions that are assumed to mirror the future shape of the 
edipemic ${ }^{5}$, complex mathematical models that attempt to mimic the transmission dynamics of the virus between and within risk groups ${ }^{6}$, and what are called backcalculation methods ${ }^{7}$. Extrapolation based on simple functions is unreliable due to the non-linear and uncertain shape of the epidemic. Transmission models contain many parameters, particularly with respect to sexual behaviour and mixing patterns, for which estimates are not available at present; hence the favoured method is back-calculation.

The principle behind it is simple. Given information on the incidence of AIDS from surveillance reports plus the probability distribution of the incubation period estimated from cohort studies, it is possible to back-calculate the number of people infected with HIV during each time period necessary to account for the observed AIDS case reports. The number of people who will be diagnosed with AIDS in the near future (2-4 years) is then projected by extrapolation based on the shape of the estimated HIV incidence curve and the incubation period distribution. Most countries rely on this method, and reports in the United States ${ }^{8}$ and the United Kingdom ${ }^{9}$ have, in general, produced reasonably reliable projections when measured against the observed outcomes. More recent calculations much more closely match observed trends (Fig. 2) whereas the early reports $(1986,1988)$ overestimated the degree of spread. The greatest uncertainty in future trends has been, and still is, among heterosexuals.

The main reason for the downwards revision in the projected incidence of AIDS in the coming few years lies in the trend revealed by the back-calculation method in the incidence of new HIV infections among homosexual and bisexual men over the past decade, where a sharp slowing down in the rate of transmission appears to have occured from about 1984. Interestingly, this trend is revealed in most developed countries and in both of the two major risk groups (homosexual men and intravenous drug users). By contrast, estimated incidence curves for heterosexuals show an upward trend over the past decade (Fig. 2).

Three factors, or some combination of them, could explain the trend in homosexual men. First, it could simply reflect changes in behaviour as a result of education and media coverage. Second, it could be a reflection of the natural pattern of the epidemic, where saturation of infection in the high-risk groups reduces the rate at which secondary cases arise. Third, it could be spurious, and a direct consequence of the assumptions underlying the back-calculation method. The first explanation is probably an important component, but direct quantitive evidence of behaviour change is limited. The second explanation must also be important, as
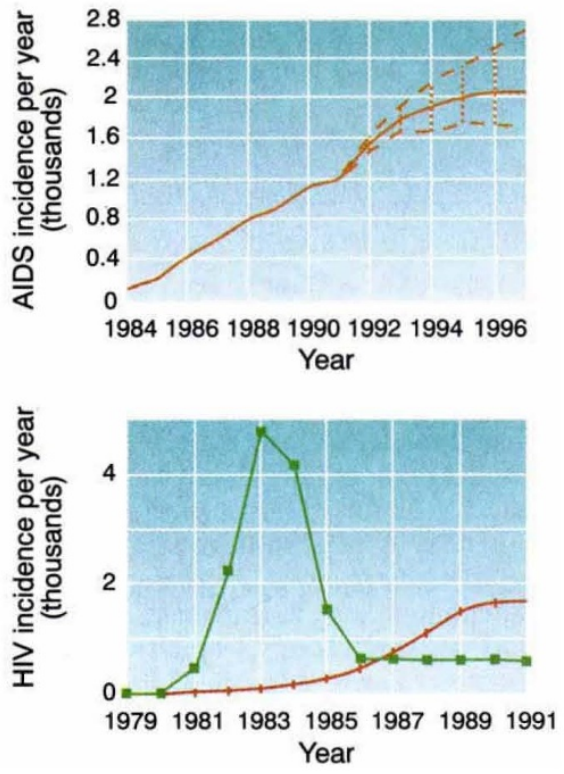

FIG. 2 Top, latest projections of AIDS incidence per annum in England and Wales in all risk groups combined ${ }^{8}$. Estimated HIV prevalance at end of 1991: lower estimate, 20,000; upper estimate, 29,050 . The solid line up to 1992 is essentially identical to the observed outcome. Beyond this date projections are based on back-calculation methods and record the planning figures (solid line) and upper and lower bounds on the projections ${ }^{9}$. Bottom graph, estimated annual incidence of new HIV-1 infection in England and Wales in homosexual/bisexual men (green) and in heterosexuals (red) from back-calculation ${ }^{9}$.

simple theory reveals a bell-shaped incidence curve for infections in a naive population, with a long right-hand tail in the absence of any change in behaviour ${ }^{10}$. The third explanation has not been explored sufficiently. First, the detailed back-projections of HIV incidence (see Fig. 2) are sensitive to the assumption of when the epidemic started. Second, the method tends to allocate most HIV infections to as early a time point as possible. Third, the full incubation period of AIDS and the fraction of those infected who will develop AIDS are unknown ${ }^{11}$. Last, the method is not suitable for assessing trends in incidence in the few years immediately preceding the last observation on AIDS incidence (the flat segment on the righthand side of the bottom graph for homosexual men in Fig. 2). Put together, these problems imply that the estimated magnitude of the HIV incidence curve may be too great early in the epidemic and too small in the most recent time frame. These methodological issues highlight the uncertainty surrounding future trends.

Simple and complex models of the transmission dynamics of HIV based on current data of the key processes (admittedly limited) suggest that, in the absence of a major breakthrough in research on drugs that halt or slow progression to AIDS as well as reducing the infectiousness of infected individuals, and without hope of vaccines to prevent infection, HIV is likely to attain an endemic state in the coming decades. The epidemic pattern before this state may be multi-peaked, perhaps with each peak separated by many years as infection passes from highvia medium- to lower-risk groups. The magnitude of the endemic stage, and the time taken to attain it, will vary between countries, as do the prevalences of other sexually transmitted infections. It appears likely that the endemic state in Europe and North America will be maintained largely within the core groups of individuals with high-risk behaviour belonging to each of the major at-risk categories. For sexually transmitted diseases in general the size of the core group varies according to the biological properties of the infection and the behavioural determinants of transmission. Endemic prevalences of other sexually transmitted diseases are not necessarily good guides to the likely extent of HIV spread in future decades. However, taking genital herpes as an example, surveys in London reveal that roughly $7-10 \%$ of pregnant women of Caucasian origin aged 35-40 have evidence of past infection ${ }^{12}$. There are obvious dangers in drawing parallels between these viruses; but these studies highlight what may be an upper bound to the potential size of the heterosexual group at risk.

The relative effectiveness of AIDS awareness and sex-education programmes for young people before they start sexual activity will obviously be important. Given the many uncertainties, a precautionary approach seems advisable, with education targeted broadly, as no one can predict to which, if any, risk group, a young teenager will eventually belong. AIDS is a dreadful disease which is invariably lethal, given current treatment options. The media therefore has a responsibility to report the facts correctly and to avoid sensationalism, whether through exaggeration or by denial.

Roy Anderson is in the Biology Department, Imperial College of Science, Technology and Medicine, London SW7 2BB, UK.

1. Chin. J., Remenyi, M-A, Morrison, F. \& Bulatoa, R. World Hith Stat. Quart. 45, 22-27 (1992).

2. Commun. Dis. Rep. 3, R1-R16 (1993).

3. Anderson, R. M. \& May, R. M. Nature 333,514-519 (1988).

4. Morbid. Wkly Rep. 41, No. RR-18, 1-29(1993).

5. Norman, C. Science 230, 1020-1 (1985)

6. Hyman, J. M. \& Stanley, E. A. Math. Biosci. 10, 415-473 (1988)

7. Brookmeyer, R. \& Gail. M.J.H. J. Am. Stat. Ass. 83 301-308 (1988).

8. Aboulker, J. P. \& Short, A. M. Lancet 341, 889-90 (1993).

9. PHLSAIDS Centre at CDSC. AIDS Projections to 1997, England and Wales. Comm. Dis. Rep. (in the press).

10. Anderson, R. M. \& May, R. M. Infectious Diseases of Humans (Oxford University Press, 1991)

11. Hendriks, J. C. M. et al. AIDS7, 231-239 (1993).

12. Ades, A. E. et al. J. epidemiol. Comm. Hith 43, 53-60 (1989).

13. WHO-EC Collaborating Centre on AIDSQuart. Rep. No. 36. 1-39 (1993).

NATURE · VOL $363 \cdot 3$ JUNE 1993 